\title{
Chirurgie orthognathique : les cas défavorables*
}

\author{
Pierre BOULETREAU** \\ CHU Lyon Sud, Chirurgie maxillo-faciale, 165 Chemin du Grand Revoyet, 69495 Pierre-Bénite Cedex, France
}

MOTS CLÉS :

Chirurgie orthognathique / Échec

KEYWORDS:

Orthognathic surgery /

Failure

\begin{abstract}
RÉSUMÉ - Le bon déroulement d'un protocole orthodontico-chirurgical nécessite un investissement sans faille de la part de ses différents acteurs, thérapeutes et patient. Lorsque le résultat obtenu n'est pas à la hauteur des espérances de changements esthétiques et/ou fonctionnels attendus, ce résultat peut être vécu comme un échec par le patient, les praticiens, voire les deux parties. Deux situations potentielles d'échec sont ici analysées : les cas défavorables et les difficultés psychologiques à accepter son nouveau visage.
\end{abstract}

\begin{abstract}
Unfavorable outcomes in orthognathic surgery. Successful completion of an orthodontic-surgical protocol requires faultless involvement of all the parties concerned, both the practitioners and the patient. When the result achieved does not match esthetic and/or functional expectations, the outcome may be seen as a failure by the patient, the practitioners or even both. Two situations comprising a potential for failure will be analyzed here: unfavorable outcomes and the psychological difficulties involved in accepting a new face.
\end{abstract}

\section{Introduction}

La prise en charge des dysmorphoses dentomaxillaires s'envisage dans l'immense majorité des cas dans le cadre d'un protocole orthodonticochirurgical. La chirurgie orthognathique s'intègre naturellement au sein de ce protocole au terme d'une phase de préparation orthodontique, et avant que la phase de finitions orthodontiques ne vienne parfaire l'occlusion. De la collaboration entre l'orthodontiste et le chirurgien nait le plan de traitement du patient dont l'objectif est idéalement d'atteindre le meilleur objectif fonctionnel et esthétique possible. Lorsque le patient a clairement été averti des objectifs envisageables, qu'il les a acceptés, et qu'ils ont été atteints, le traitement est un succès.

Dans un certain nombre de cas cependant, le résultat peut être vécu comme un échec thérapeutique par le patient, les praticiens, voire les deux parties.

\footnotetext{
* Retrouvez l'intégralité de la communication orale de P. Bouletreau le samedi 14 mai 2016 lors de la $88^{e}$ réunion scientifique de la SFODF à Marseille.

** Auteur pour correspondance : pierre.bouletreau@chu-lyon.fr
}

Nous ne traiterons pas des échecs techniques purs (défaut de repositionnement condylien par exemple) ou des complications chirurgicales responsables de mauvais résultats fonctionnels (infection et échec de l'ostéosynthèse...) ou esthétiques (encoche préangulaire marquée liée à une résorption osseuse ou fistule cutanée). Deux situations méritent d'être plus finement analysées : les cas défavorables et les difficultés psychologiques à accepter son nouveau visage.

\section{Les cas défavorables}

Dans un certain nombre de cas, les conditions anatomiques squelettiques, dentaires ou parodontales ne permettent pas d'atteindre le double objectif fonctionnel et esthétique espéré et une solution de compromis thérapeutique doit être acceptée.

Les discordances esthético-fonctionnelles sont un exemple typique de cas défavorables. Il s'agit par exemple de patients en classe III qui requièrent à l'évidence un ample mouvement squelettique de propulsion maxillaire afin de corriger un défaut de volume de l'étage moyen, mais chez qui le surplomb négatif pré-chirurgical n'est que de 
quelques millimètres... Le résultat esthétique final sera sub-optimal et le patient conservera un profil esthétique de classe III. Dans un autre registre, les patients en classe II avec progénie relative sont un autre exemple classique de cas défavorable, et il est extrêmement difficile de masquer un menton proéminent lorsqu'une avancée mandibulaire est programmée pour répondre à l'objectif fonctionnel. De même, l'esthétique de la région nasolabiale est parfois un frein à la propulsion et/ou à l'impaction maxillaire lorsqu'il existe un angle nasolabial ouvert et une pointe du nez relevée, tout comme l'empâtement de la région sous-mandibulaire interdit tout geste de recul mandibulaire... Ces diverses situations devront être détectées par le praticien très en amont de la phase thérapeutique afin que le résultat final ne soit pas vécu comme un échec par le patient, mais bien comme le meilleur compromis que ses praticiens pouvaient lui proposer. De la même manière, les conditions dentaires et parodontales sont sources de contraintes thérapeutiques pour l'orthodontiste qui devra parfois accepter un résultat occlusal sub-optimal afin de conserver la denture.

\section{Accepter son nouveau visage}

La dimension psychologique de la chirurgie orthognathique, longtemps mal connue voire ignorée, est actuellement une des clés du succès thérapeutique. Lorsque le changement esthétique est voulu par le patient et que le résultat est à la hauteur de ses attentes, tout va bien. Les difficultés émergent lorsque le résultat esthétique n'est pas celui espéré par le patient, qu'il s'agisse d'un défaut du plan de traitement ou d'un cas défavorable. Mais la situation la plus difficile en termes d'acceptation de son nouveau visage concerne le patient, adulte souvent, qui subit un changement esthétique non désiré.

Cette situation est rencontrée de plus en plus fréquemment chez nos patients adultes dont le motif de consultation unique sinon principal est la crainte du déchaussement dentaire. Ces patients qui viennent avec une demande purement fonctionnelle se voient imposer un changement esthétique de leur visage qui, même s'il n'est pas majeur, est souvent mal vécu.

\section{Exemple de cas défavorable}

Patient présentant une classe III par rétromaxillie. En fin de préparation orthodontique, le surplomb

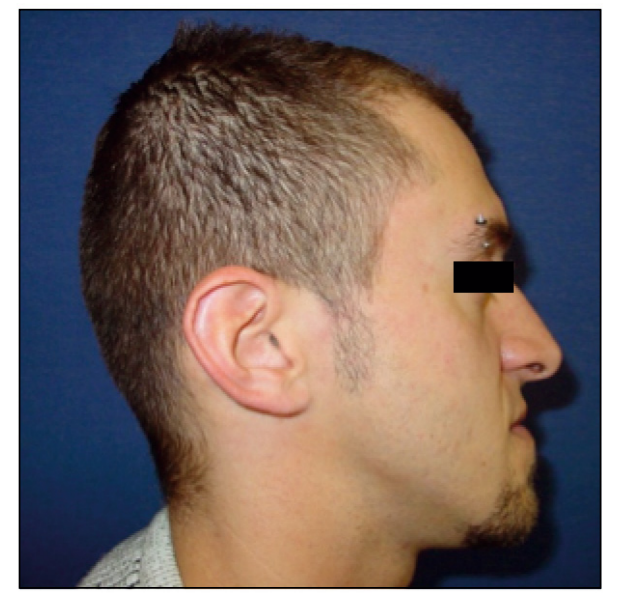

Figure 1

Vue de profil pré-opératoire.

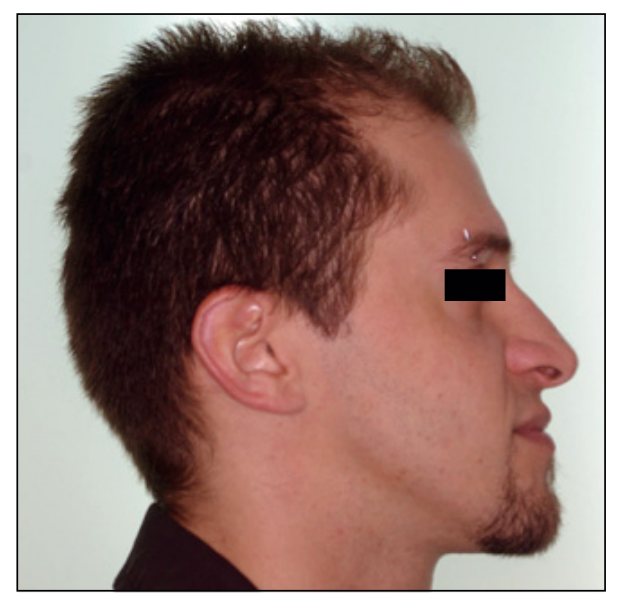

Figure 2

Vue de profil post-opératoire (8 mois).

négatif est de moins de $4 \mathrm{~mm}$. Une ostéotomie de Lefort I de propulsion a été réalisée (Figs. 1 à 4).

Commentaire : Le résultat esthétique postopératoire est imparfait car le patient garde un profil esthétique de classe III dont il s'est plaint. Une amplitude chirurgicale de 6 à $8 \mathrm{~mm}$ aurait été nécessaire afin d'obtenir un bon résultat esthétique. Un second temps opératoire de greffe osseuse d'apposition dans les secteurs para-latéronasaux a permis d'améliorer le résultat esthétique.

\section{Conclusion}

Si nul n'est à l'abri d'un aléa thérapeutique, une bonne connaissance des situations défavorables permet de limiter au maximum les risques d'échecs d'un protocole orthodontico-chirurgical. 


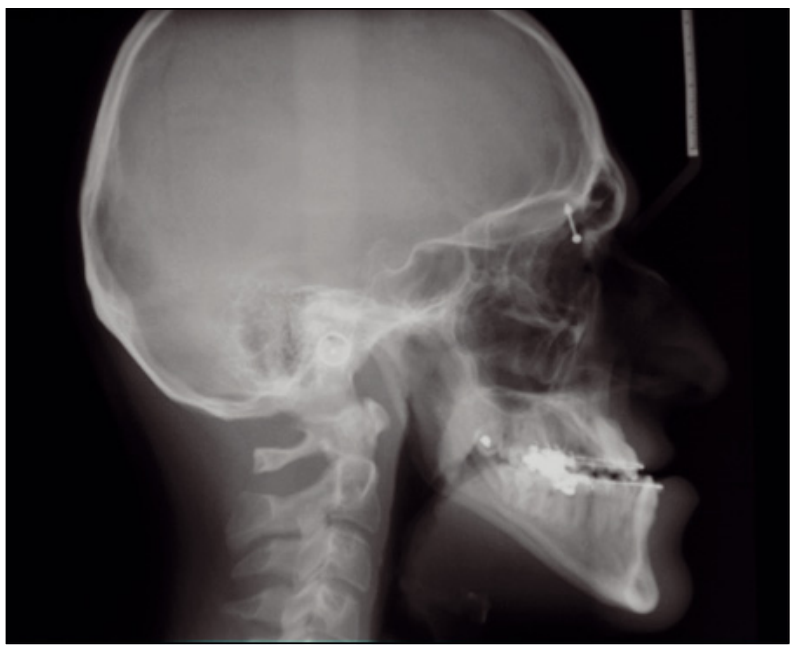

Figure 3

Téléradiographique de profil pré-opératoire.

Linformation claire et honnête du patient quant aux objectifs réalisables reste le principe fondamental à respecter pour éviter les situations conflictuelles, et

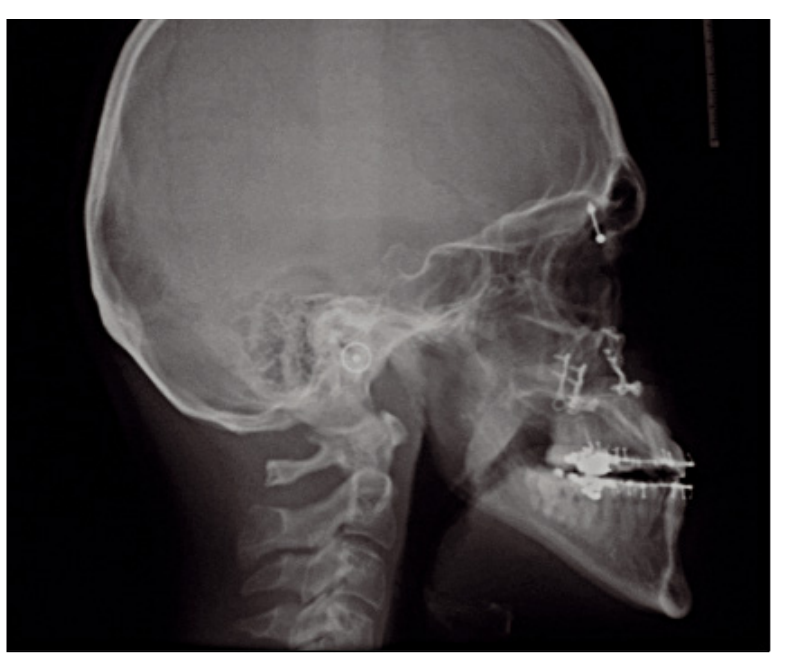

Figure 4

Téléradiographique de profil post-opératoire.

plus particulièrement dans les situations présentées qui peuvent être vécues comme des échecs par les patients. 